\title{
Remote Interpreting: Platform Testing in a University Setting
}

\author{
Francesco Saina \\ ${ }^{1}$ SSML Carlo Bo, Rome and Bari, Italy \\ f.sainaessmlcarlobo.it
}

\begin{abstract}
This work is based on the testing of a remote interpreting (RI) delivery platform conducted a year before the disruptive COVID-19 pandemic outbreak, and aimed at assessing the use and experience of such systems in a university setting. A survey was administered to the different groups of users (interpreters, audience, and speakers) involved in two tests to collect their responses and remarks, and assess trends and perceptions in their experience. According to the findings of the research project, the RI environment was already considered to be an indisputable yet burgeoning resource for conference settings with potential convenience and benefits for each group of users. However, participants' remarks early suggested that all the parties involved in the industry need to collaborate to effectively improve and enhance such services. Specific training on RI modalities would also appear to be increasingly necessary for interpreters to adapt to emerging working conditions and meet a thriving demand - and training institutions would ever more have to offer adequate solutions, while this technological shift also requires receptiveness and adaptability to an abruptly diversifying and evolving profession.
\end{abstract}

Keywords: Interpreting Technology, Distance Interpreting, Remote Interpreting.

\section{Introduction}

As in almost any professional field and communication setting, technology has taken a leading role in interpreting too. Over the last few years, remote interpreting (RI) specifically has become an ever-increasing modality being used in conference interpreting [14] — and in the time of global movement restrictions imposed by the COVID-19 pandemic, even the only modality enabling working continuity for professionals worldwide.

This work tests an RI delivery platform and aims at assessing the use and experience of such systems in a university setting - before their widespread use for teaching purposes following the Coronavirus outbreak.

After using the RI platform for two tests held at UNINT University in Rome, Italy, in April 2019, a survey was administered to the different groups of users involved in the proceedings (interpreters, audience, and speakers) to collect their responses and 
remarks, and thus assess their experience with the use of such tools in an academic environment.

\subsection{Research Methodology}

The events took place in the 'Aula Magna' at UNINT University, where speakers and the audience gathered. A few participants followed the events from remote locations. A remote interpreting 'hub' was located in a classroom on the upper floor of the building, equipped with standard interpreting booths.

Interpretation for the events was provided by teams of volunteer interpreting students in the last year of their Master's Degree, with different levels of previous working experience. Only a limited number of students interpreted for both conferences.

After each of the two conferences, all the participants in the events (interpreters, audience, and speakers) were given a printed individual survey to fill out (remote listeners were sent a digital copy). Answers were then rigorously converted and entered into a specific digital database for a complete and accurate analysis.

Indeed, the survey was considered to be the most suitable instrument for the scope of this research, as it is a comprehensive 'means for gathering information about the characteristics, actions, or opinions of a large group of people' [15], and in accordance with the ultimate aim of any survey research, that of advancing academic knowledge in a scientific field [12].

Statistical Methodology. The various surveys contained 23 to 28 items, composed of closed-ended questions - either multiple-choice or yes/no. To collect more qualitative findings from each participant in the test and explore both general and individual attitudes, most questions were followed by a blank text box which the respondent could use for writing additional specifications or remarks and in-depth motivations.

A limited number of items invited the respondent to indicate a value on a Likert scale of 1 to 5 . A final blank space for any further voluntary observation, comment, or personal impression was offered at the very end of the survey.

Clearly, personal values attributed by individual respondents to given parameters are not absolute and necessarily arbitrary. Therefore, average (or mean) values for all answers were calculated and collected to provide more representative results and compensate for such personal differences in grading.

Of course, it is also necessary to take into consideration the relatively limited size of the sample (i.e. groups of users participating in this research) when evaluating the accuracy of the results presented in this experimental study. A total amount of 98 surveys were administered to the three groups of users (interpreters, audience, and speakers) over the two conferences and 66 were returned.

Intuitively (but also according to the statistical notion of standard error), tests involving a larger sample would offer more representative estimations. 


\subsection{Survey Sample and Structure}

Each group of users participating in the surveys was designed differently.

Interpreters were selected by the university's professors for the first test (a conference specially organized for the purpose of this experiment), while voluntary candidates were involved in the second event (and already planned conference with the use of the platform being proposed after the successful experience of a few days earlier).

Speakers participated in the two conferences following invitations from the organizing teams of each event, whereas audience members were both attendees spontaneously interested in the events' topics and specially invited guests.

According to the purposes of this study, the surveys were structured in three main sections.

The first aimed at collecting background information about the user, such as familiarity with conference interpreting settings, possible previous experience with RI, and self-assessed technological expertise.

The second part consisted of more detailed questions about the use of the platform during the event/test (the device employed to access it, evaluation of image and sound quality, RI-related issues such as concentration, sense of participation, fatigue, use of the features offered by the platform, etc.)

In the third and final section, perceptions, and opinions on RI both in general and in relation to traditional simultaneous interpretation were asked about.

\subsection{Participants Data}

Basic biographical data was collected from survey participants. However, given the reasonably lower number of speakers involved in the two conferences, they were not asked to indicate any personal information for granting their anonymity.

$89 \%$ of participants from the remaining two groups examined (interpreters and audience) were female and $11 \%$ were male.

As previously mentioned, interpreters were all UNINT students in the final year of their Master's Degree in Conference Interpreting, thus their average (mean) age was 23.56 , with standard deviation (the measure of dispersion of the values from the average value, i.e. the mean) 0.84 .

Three language teams (English, Italian, and Spanish) with a total amount of 14 interpreters worked during first event, whereas 18 interpreters (offering two additional languages: French and Portuguese) provided their service for the second test. Only six interpreters participated in both events.

All the interpreters taking part in the two events were extremely collaborative and completed the surveys.

An approximate total amount of 80 people attended the two events held at UNINT University where the platform testing was carried out. 58 attendees listened to the interpreting service provided via the RI platform and, at the end of the events, they were handed the printed survey and encouraged to fill it out-while they were also reminded that participation in the survey was completely voluntary. 
32 audience members returned the completed survey. Respondent audience age ranged from 17 to 47 years $(M=24.44, S D=5.72)$.

The data obtained from the speakers' group is significantly limited as only two complete surveys were returned.

Since most of the speakers were preeminent professors and distinguished academics (alongside a few foreign guests speaking during the second event), it is to be taken into account that, despite their willingness, their activities can often limit their possibilities of fully participating in projects like this test. However, the data collected represents the perceptions and opinions on the RI platform of two qualified speakers and their considerations can be valuable even so to this research.

Since the floor source input (i.e. the presentations delivered by the speakers) was transmitted only from one computer, managed and controlled by UNINT's technical staff, speakers accessed the platform from their devices by using the audience token (access code), therefore in the survey they were mainly asked questions on their listening experience.

Survey and participants data is summarized in Table 1 below.

Table 1. Survey and participants data.

\begin{tabular}{lllll}
\hline Group & Administered & \multicolumn{2}{l}{ Returned } \\
\hline Interpreters & 32 & & 32 & \\
Audience & 58 & & 32 & \\
Speakers & 8 & & 2 & \\
Total & 98 & & 66 & \\
\hline \multicolumn{5}{c}{} \\
Gender (interpreters + audi- & $\mathbf{\%}$ & Age (group) & Mean & SD \\
ence) & & & & \\
\hline Female & 89 & Interpreters & 23.56 & 0.84 \\
Male & 11 & Audience & 24.44 & 5.72 \\
\hline
\end{tabular}

\section{$1.4 \quad$ Pilot Testing}

On March 29, 2019, as suggested by Levy \& Lemeshow's indications [6], a pilot test with survey administration to a restricted sample was conducted to validate the survey and assess the intelligibility and accuracy of the questions. Participants involved in this pilot survey were not the same as those who would participate in the actual test.

Two days before the first experiment, a final pilot test aiming at verifying the functioning of the platform and all technical requirements was conducted with all the interpreters who would participate in the research experiment, with the purpose of making them begin familiarizing with the system. 


\section{$2 \quad$ Findings}

Due to the impossibility of presenting here the full report of the data collected from the surveys administered to the three groups of users, the respondents' most significant answers (and the remarks they added in the available blank spaces) will be analyzed and discussed to observe the scenario offered by the surveys, assess the results of this test, and outline general trends emerging from the users' experience.

\subsection{Interpreter Survey Results}

Despite interpreters testing the platform were students, survey results show that the majority among them (75\%) had already had work experience, some of them (19\%) even with RI.

Evaluations on video and audio quality were generally positive, but they show that there is still room for improvement of both, on the one hand, the quality of the video feed that event organizers can provide and, on the other hand, the reliability of the signal that the platform can ensure.

Further remarks expressed by the interpreters in the blank spaces available in the survey suggest that a video-mediated view of the speaker is only perceived as an additional asset when a zoomed-in view-which allows seeing nonverbal communication elements, gestures, and lip movements - is offered, thus providing interpreters with a better view than what they could usually see from an on-site booth.

Since remote interpreting is usually reported to have an impact on the interpreter's sense of fatigue and to produce an increased cognitive effort compared to an assignment in traditional simultaneous interpreting equivalent in time [4, 7, 2], interpreters were suggested to work in 15 -minute turns, slightly shorter than average simultaneous interpreting turns - in accordance with recommendations by various guidelines and academic publications on RI [17, 2].

However, most of them seemed not to experience any increased mental and/or physical fatigue (91\%) nor distraction (75\%) caused by the use of RI, and this could be partly attributed to the fact that, in their training courses, they were already used to practicing with videos and in RI-like conditions.

Indeed, almost all of them (78\%) perceived their performance the same as compared to traditional interpreting conditions - although half of them reported the platform itself to hinder their performance to a certain extent.

The notions of 'presence' and sense of participation in the communication event are also frequently mentioned when discussing RI in the academic and professional communities $[9,10,8,16]$.

Nevertheless, $78 \%$ of interpreting students - who are possibly more used to interacting and communicating in virtual environments - did not feel alienated due to the fact that they were not located in the main conference room.

A couple of them - among the less experienced - even added in the blank spaces for personal remarks that being in a remote location helped them cope with the stress and pressure caused by an on-site working environment, ultimately benefitting their performance. 
Other respondents added that being in the hub created a 'friendly and cooperative environment' among the interpreting team, which helped interpreters 'feel more comfortable and confident' (comments reported from the interpreters' surveys).

Furthermore, they did not seem to perceive the video-mediated view of the speaker and the absence of direct visual feedback from the audience to have a significant impact on the quality of their performance.

Almost no interpreter (3\%) used the event chat feature offered by the platform, thus proving that not always the availability of more features coincides with a better environment for the interpreter, at least when boothmates are co-located as in this case, since the interpreting task requires extreme concentration and adding further elements could occasionally increase the cognitive load and interfere with the interpreter's attention and performance.

Besides, the relay feature could appear seemingly more intuitive than its equivalent in traditional consoles at first (since it is also set before the beginning of the event), but most interpreters (67\%) did not perceive any remarkable difference in its use.

One respondent suggested creating 'a keyboard shortcut, e.g., the spacebar' to make the use of the 'mute' microphone feature easier and more direct, without the need to use the mouse cursor.

Another interpreter reported the inconvenience of having to log in again when the web page is refreshed, and having the laptops inside the booth with less space for personal belongings and working materials (e.g., printed documents and glossaries) was mentioned as another element of discomfort.

$78 \%$ of interpreters accepted favorably a few additional conditions required by the platform (such as the use of personal devices, the possibility of running out of charge, the need to download the mobile app to listen to their colleagues' performances), without considering that an inconvenience.

Moreover, probably by virtue of their familiarity with information and communication technologies (ICTs), 91\% of interpreting students found the use of the platform intuitive and immediate, and therefore did not need any additional informative material besides a guide they were provided with by the platform company and a whole morning to test the platform two days before the experiment.

A few of them $(22 \%)$ complained about not having received presentations and materials shown to the audience by the speakers beforehand, since these are not always sharply visible in an RI setting, even when a dedicated screen is provided.

However, slightly more than half of them $(56 \%)$ believe that specific training for learning how to use and work with such tools is necessary, at least (in the interpreters' own words) 'a couple of lessons' should be introduced into regular courses to 'familiarize with the use of such systems', and 'dedicated preparatory sessions' before any assignment are considered to be 'essential to verify the functioning of the platform' and feel 'confident on the day of the event'.

One respondent suggested the use of video tutorials for learning how to use the platform faster and more immediately.

The need for 'available adequate equipment' when practicing with such systems and when preparing for an RI assignment was also highlighted. 
Above all, most of them expressed the awareness of the fact that such tools may have a preeminent role in 'the future of the interpreting profession' and therefore 'training courses should take that into consideration', since being able to master these systems can be 'a valuable asset on the market'.

The importance of familiarizing with the platform is also supported by the fact that 5 out of the 6 interpreters who participated in both tests found the second experience to be better than the first on the whole - the one remaining considered it 'the same'.

Table 2 outlines the most significant quantitative data collected from interpreters.

Table 1. Quantitative data from the interpreters' surveys.

\begin{tabular}{llll}
\hline Interpreters & Yes (\%) & No (\%) & N/A (\%) \\
\hline Previous work/internship interpreting experience & 75 & 25 & $/$ \\
Previous experience with RI & 19 & 81 & $/$ \\
Increased mental and/or physical fatigue & 9 & 91 & $/$ \\
Increased distraction & 22 & 75 & 3 \\
Platform-related obstacles & 50 & 47 & 3 \\
Feeling part of the event & 78 & 9 & 13 \\
Performance affected by lack of direct visual feed- & 3 & 94 & 3 \\
back & 3 & 97 & $/$ \\
Use of the event chat & 22 & 78 & $/$ \\
Platform conditions as inconvenience & 91 & 9 & $/$ \\
Platform is easy-to-use, intuitive, and immediate & 22 & 78 & $/$ \\
Need more materials & 56 & 44 & $/$ \\
Specific training for RI & & & \\
\hline
\end{tabular}

\subsection{Audience and Speaker Survey Results}

Moving the discussion to the data collected from the audience, in the first place it must be underlined that most attendees listening to the interpreting service were other university students in conference interpreting, alongside professors, researchers and professional interpreters of UNINT's academic community, and a few participants from other faculties or even institutes.

Listeners followed the event both from the conference room and remote locations, with a couple of the former also moving from the event venue to other positions inside or outside the university while bringing their personal devices with them, and a limited group (13\%) participating in both events where the platform was tested.

Half of them had never heard about RI before this test and only very few of them $(9 \%)$ had participated in other events where RI services were provided. This indicates that even in events attended by the interpreting community, RI had not become a prevalent solution yet, but most of them $(60 \%)$ only rarely take part in events where interpretation services of any kind are offered - or at least they do not need interpretation during the events they usually attend. 
$62 \%$ of them accepted downloading the app on their mobile devices to listen to the interpretation service, while those who accessed the platform via its webpage without downloading any software could also follow the video of the event.

Overall evaluations on video and audio quality (and synchronicity between what was happening in the event venue and the transmission of the signal) from the audience were positive again, but encouraged improvements to definitely make the RI platform an optimal solution. Including the video feed and the event chat on the mobile app too was mentioned by a few audience members as a recommend upgrade.

A considerable majority of respondents did not experience any increased fatigue or stress $(88 \%)$, distraction $(81 \%)$, and obstacle $(72 \%)$ due to the platform, thus indicating a general positive experience for the users.

Saving time usually dedicated to the distribution and return of the receiving devices and headphones was also remarked by three respondents as an advantage of being able to use personal devices. Additional remarks praised both app and web userfriendly interfaces and the easy selection of language channels.

One of the remote listeners defined 'being able to follow the conference from a distant location while listening to the interpretation service in different languages' as 'revolutionary and simple at the same time'. Two different remote listeners remarked increased distraction since they were following the event from home instead.

Three respondents praised the possibility to continue listening to the audio feed also after exiting the app, and therefore being able to simultaneously use their mobile devices for separate needs. Nevertheless, two different respondents mentioned this same possibility as leading them to distraction.

Dissenting respondents agreed on some complaints about the platform, i.e., rapidly running out of charge on their personal devices (claimed by eight respondent audience members and also mentioned by a few interpreters listening to their colleagues via their mobile devices), and listening to the audio signal from their device speakers even after disconnecting their headphones (reported twice as an inconvenience). One respondent only also signaled slowness when changing language channels.

Additionally, features like a general event chat appear not to make a significant difference in the user experience, unless specific and individual chat options are offered.

The main quantitative data obtained from attendees is displayed in Table 3 below.

Table 1. Quantitative data from audience surveys.

\begin{tabular}{llll}
\hline Audience & Yes $(\%)$ & No $(\%)$ & N/A $(\%)$ \\
\hline Awareness of RI before the test & 50 & 47 & 3 \\
Participation in events with RI & 9 & 91 & $/$ \\
Increased fatigue or stress & 12 & 88 & $/$ \\
Increased distraction & 19 & 81 & $/$ \\
Platform-related obstacles & 25 & 72 & 3 \\
Use of the event chat & 6 & 94 & $/$ \\
Platform conditions as inconvenience & 25 & 69 & 6 \\
Platform is easy-to-use, intuitive, and immediate & 91 & 6 & 3 \\
\hline
\end{tabular}


Finally the two speakers, albeit indicating a high degree of familiarity with ICTs, were at their first experience with an RI platform.

Members of this users group expressed moderate positive evaluations on audio quality and synchronicity and an overall favorable judgment on the platform - still taking into account that they had little direct involvement in the use of the platform as speakers since the technical staff entirely prepared and managed it for them.

However, their experience as users of the platform for listening to the interpreted audio did not significantly hinder their speaking task nor increase their stress or distraction in any reported way.

\section{Conclusion}

The objective of this research was to investigate the use and observe the experience of a remote simultaneous interpreting platform in a university setting. This study did not aim at evaluating the platform itself nor expressing an ultimate judgment on the implementation of remote interpreting as an accomplished working modality.

The purpose of the project was rather to collect the users' perceptions and remarks and assess trends in their experience, by testing a professional platform on a limited but representative sample of participants, composed of interpreting students, professional and non-specialized audience, and speakers.

\subsection{Discussion}

Interpreting students testing the platform are clearly short of extensive experience and competency to express a more accurate and comprehensive evaluation on RI advantages and disadvantages, however they also are the future professionals who will encounter these tools in their working environments more than any previous generation, thus collecting their impressions and inclinations towards such systems is a valuable standpoint. Results and tendencies emerging from their answers suggest a remarkably responsive and receptive approach, and openness to innovation and evolution in the profession.

Previous experiments on distance interpreting reported that professional interpreters often find difficulties in embracing RI solutions: since they are used to automated processing when performing their tasks in traditional interpreting conditions, they are therefore hindered when trying to accommodate new variables [8].

Notwithstanding, large-scale medical examinations did not find any evidence of additional stress, and a performance evaluation assessed that remote interpreters' outputs are slightly inferior compared to those of on-site interpreters, yet not enough to achieve statistical relevance [7, 13].

Furthermore, it is not to be forgotten that already in the middle of the twentieth century, most prominent consecutive interpreters refused to adapt to the then recentlyborn simultaneous modality [5]. As a matter of fact, Moser-Mercer [8] had already perceived that interpreters with years of professional practice 'may be less likely to 
adapt to a new working environment than less experienced colleagues who may exhibit a greater degree of adaptive expertise'.

Audience and speakers' responses show that RI has achieved offering a satisfactory overall experience by now. Nevertheless, there is still room for maneuver and collaboration among all the parties involved in such evolution appears to be the best way to developing and implementing increasingly better solutions - and RI delivery platforms have regularly and relentlessly been updating their systems during the COVID-19 emergency to keep pace with constantly evolving needs.

The technological development has completely shifted the paradigms of society and work as a whole: professionals in any field are adapting to new working modalities and conditions and, since communication patterns also are considerably evolving, the interpreting profession too will inevitably be involved in such virtual revolution and will diversify accordingly $[9,1,11,3]$.

The adaptability of junior interpreters clearly needs to be associated with rigorous and in-depth preparation. The need for specific training on RI systems during interpreting courses and the availability of adequate equipment in training institutions were both expressed by interpreting students participating in this test, thus providing support to what Ziegler and Gigliobianco [18] had already acutely underlined.

Most attention should be paid to the effect of additional practice and familiarization with the platform on the five interpreters who participated in both events and already considered the second experience better than the first.

\subsection{Further Research}

The aforementioned outcome motivates the proposition of the need to carry out more than one single experiment with the same participants (and on bigger samples, too) taking into account another parameter: time variation.

This could allow a comparison between subsequent sessions and further examination of new criteria, such as the evolution of trends over time, the consequences of increasing expertise of the users with the platform, or potential changes in the perception of the performance and the overall service.

Moreover, during the tests carried out for this research, same-language colleagues have been working in the same booth. Therefore, an additional challenge to be explored would be turn handover and communication between boothmates when interpreters are not placed in the same location.

The COVID-19 pandemic, besides its dramatic impact on global health, economy, and society, is also an unprecedented challenge in the history of interpreting, questioning several tenets of the profession itself. It will undoubtedly mark RI as one of the main subjects in the interpreting research field over the next years, as it deserves and offers space for extensive supplementary exploration.

The outcome of this work may provide training institutions with insights and indications on how to implement RI tools in their environment after the Coronavirus emergency when shaping the post-pandemic academic scenario, and the considerations in the last paragraphs could pave the way to only a few possible paths for additional research interest and further investigation. 


\section{References}

1. AIIC (Private Market Sector Standing Committee): Remote simultaneous interpreting: Time to start a dialogue (2019), https://aiic.net/p/8755, last accessed 2019/10/09.

2. Braun, S.: Remote interpreting. In: Mikkelson, H., Jourdenais, R.: The Routledge handbook of interpreting. Routledge, New York, NY (2015).

3. European Commission (Directorate General for Interpretation): Interpreting platforms: Consolidated test results and analysis (2019), https://ec.europa.eu/education/knowledgecentre-interpretation/sites/kci/files/interpreting_platforms_consolidated_test_results_and_analysis_-_def.pdf, last accessed 2021/05/25.

4. European Parliament (Interpretation Directorate): Report on remote interpretation test. 22 25 January 2001. Brussels (2001), http://www.europarl.europa.eu/interp/remote_interpreting/ep_report1.pdf, last accessed 2021/05/25.

5. Gaiba, F.: The origins of simultaneous interpretation: The Nuremberg trial. University of Ottawa Press, Ottawa (1998).

6. Levy, P.S., Lemeshow, S.: Sampling of populations: Methods and applications. 4th edn. Wiley, Hoboken, NJ (2008).

7. Moser-Mercer, B.: Remote interpreting: Assessment of human factors and performance parameters (2003), last accessed 2019/10/09.

8. Moser-Mercer, B.: Remote interpreting: The crucial role of presence. Bulletin suisse de linguistique appliquée 81, 73-97 (2005).

9. Mouzourakis, P.: That feeling of being there: Vision and presence in remote interpreting (2003), https://aiic.net/p/1173, last accessed 2019/10/09.

10. Mouzourakis, P.: Remote interpreting: A technical perspective on recent experiments. Interpreting 8(1), 45-66 (2006).

11. Olsen, B.S.: Remote interpreting: Feeling our way into the future. The ATA Chronicle 46(3), 14-16 (2017).

12. Pinsonneault, A., Kraemer, K.L.: Survey research methodology in management information systems: An assessment. Journal of Management Information Systems 10, 75-105 (1993).

13. Roziner, I., Shlesinger, M.: Much ado about something remote: Stress and performance in remote interpreting. Interpreting 12(2), 214-247 (2010).

14. Seeber, K.G.: Distance Interpreting survey: Answers to seven questions (2018), http:// members.aiic.net/p/8640, last accessed 2019/10/09.

15. Tanur, J.M.: Advances in methods for large-scale surveys and experiments. In: McAdams, R., Smelser, N.J., Treiman, D.J.: Behavioral and social science research: A national resource (Part II). National Academy Press, Washington, DC (1982).

16. Viaggio, S.: Interpretation: Theory and practice (2019), http://sergioviaggio.com/?p=4330, last accessed 2021/05/25.

17. Wisconsin Court System: Guidelines for using telephonic interpreting in court (2011), https://www.wicourts.gov/services/interpreter/docs/telephoneinterpet.pdf, last accessed 2021/05/25.

18. Ziegler, K., Gigliobianco, S.: Present? Remote? Remotely present! New technological approaches to remote simultaneous conference interpreting. In: Fantinuoli, C. (ed.): Interpreting and technology, pp. 119-139. Language Science Press, Berlin (2018). 